\title{
The level of stress and body self image perception in adolescents with idiopathic scoliosis
}

\author{
Edyta Kinel $^{1 *}$, Anna Podolska-Piechocka ${ }^{1}$, Tomasz Kotwicki ${ }^{2}$, Przemyslaw Lisinski ${ }^{1}$ \\ From 11th International Conference on Conservative Management of Spinal Deformities - SOSORT 2014 \\ Annual Meeting \\ Wiesbaden, Germany. 8-10 May 2014
}

\section{Background}

The level of stress and body self image perception in girls suffering from adolescent idiopathic scoliosis (AIS) is the object of interest of the professionals.

\section{Aim}

Evaluation of the stress level in adolescents with AIS and their self image perception.

\section{Design}

Observational study. It involved 82 adolescents, ages ranging between 11.0 and 16.0 years, all with IS with Cobb angle between 20-45 degrees. Adolescents were wearing the Chêneau orthosis, (more than 3 months, at least 12h per day). The age of examined group was $13.5 \pm 1.6$ years.

\section{Methods}

The following were used in the evaluation: Bad Sobernheim Stress Questionnaire (Brace and Deformity), Trunk Appearance Perception Scale (TAPS), and Knee - Feet Perception Scale (KFPS). The BSSQ Brace estimates the stress scoliosis patients have whilst wearing brace, the BSSQ Deformity estimates the stress involved with body deformation. Minimal points number equals 0 (the greatest stress), maximal 24 (the least stress). The TAPS evaluates subjective impression of trunk deformity from 3 viewpoints: looking toward the front, back, and with the patient bending over. Minimal points number equals 1 (the biggest deformity), maximal equals 5 (the least deformity). KFPS estimates subjective impression of knee joints and feet position. Minimal points number equals 1 (the biggest deformity), maximal equals 3 (the least deformity).
Additionally results of TAPS and KFPS were compared with clinical examination.

\section{Results}

Cobb angle was $31.0 \pm 8.1$ degrees. The BSSQ Brace and Deformity median was 13 , the TAPS median of the total score was 4, the KFPS median of the total score was 3. There were significant differences between Cobb angle and TAPS (AIS) and Cobb angle and TAPS clinical examination $(\mathrm{p}<0.001)$.

\section{Conclusions}

Conservative treatment does not severely impact on the level of stress and the self image of adolescents with idiopathic scoliosis.

\section{Authors' details}

'Department of Rheumatology and Rehabilitation Clinic of Rehabilitation, University of Medical Sciences, Poznan, Poland. ${ }^{2}$ Department of Paediatric Orthopaedics and Traumatology, University of Medical Sciences, Poznan, Poland.

Published: 4 December 2014

\section{References}

1. Botens-Helmus C, Klein R, Stephan C: The reliability of the Bad Sobernheim Stress Questionnaire (BSSQbrace) in adolescents with scoliosis during brace treatment. Scoliosis 2006, 1:22.

2. Bago J, Sanchez-Raya J, Sanchez Perez-Grueso FJ, Maria Climent JM: The Trunk Appearance Perception Scale (TAPS): a new tool to evaluate subjective impression of trunk deformity in patients with idiopathic scoliosis. Scoliosis 2010, 5:6.

doi:10.1186/1748-7161-9-S1-062

Cite this article as: Kinel et al:: The level of stress and body self image perception in adolescents with idiopathic scoliosis. Scoliosis 2014 9(Suppl 1): O62. 